Brian D. Southern, MD

Departments of Pulmonary Medicine and Inflammation

and Immunity, Cleveland Clinic; Assistant Professor, Cleveland

Clinic Lerner College of Medicine of Case Western Reserve

University, Cleveland, $\mathrm{OH}$

\title{
Patients with interstitial lung disease and pulmonary sarcoidosis are at high risk for severe illness related to COVID-19
}

\section{Updated June 2, 2020}

\section{ABSTRACT}

Analyses of COVID-19 patients with preexisting interstitial lung disease (ILD) or pulmonary sarcoidosis is lacking but registries are ongoing. Treatment of COVID-19 in patients with underlying ILD or sarcoidosis may include hospital admission, possible drug treatment, caution with steroids, and avoidance of mechanical ventilation in acute exacerbation of ILD. Patients with COVID-19 respiratory illness are at risk for developing ILD.

\section{ILD AND COVID-19}

About 2\% of patients with coronavirus disease 2019 (COVID-19) have concomitant pulmonary disease, which is associated with worse outcomes. ${ }^{1}$ The US Centers for Disease Control and Prevention (CDC) says that those at higher risk include those with any chronic lung disease or moderate to severe asthma. ${ }^{2}$ Mechanistically, this makes sense, since coronavirus gains entry into type II pneumocytes through the angiotensin-converting enzyme II receptor. ${ }^{3}$ Therefore, infected patients with any underlying chronic lung disease, especially those that result in pulmonary fibrosis, would be expected to be at higher risk of severe complications. (Other comorbidities associated with poor outcomes are cardiovascular disease and diabetes. ${ }^{1}$ ) There is no evidence to suggest that patients with pulmonary or systemic sarcoidosis who do not have pulmonary fibrosis are at increased risk of severe complications.

The statements and opinions expressed in COVID-19 Curbside Consults are based on experience and the available literature as of the date posted. While we try to regularly update this content, any offered recommendations cannot be substituted for the clinical judgment of clinicians caring for individual patients.

doi:10.3949/ccjm.87a.ccc026
As yet, there have been no specific analyses of COVID-19 patients with preexisting interstitial lung disease (ILD) or pulmonary sarcoidosis, but registries are ongoing.

\section{Histopathology}

In one of the first pathology reports of a patient who died of COVID-19 respiratory failure, ${ }^{4}$ the lungs demonstrated desquamation of pneumocytes, hyaline membrane formation, and pulmonary edema, ie, the histopathologic picture of diffuse alveolar damage seen in acute respiratory distress syndrome (ARDS). Similar findings were seen in the first two reported autopsy cases in the United States. ${ }^{5}$ This is the pathologic pattern most commonly seen in acute exacerbations of ILD.

\section{Could COVID-19 trigger acute exacerbations of ILD?}

Although the exact mechanism of acute exacerbation of ILD is not fully understood, the current belief is that it can be caused by numerous triggers, including infection, or it can be idiopathic. ${ }^{6}$ It is likely that respiratory infection with COVID-19 could trigger an exacerbation of underlying ILD and result in poor outcomes. There is similar concern regarding the possibility of exacerbation in patients with sarcoidosis, especially those with fibrotic manifestations. ${ }^{?}$

\section{How has the COVID-19 pandemic affected care of patients with ILD?}

A longstanding issue in the field has been delayed ILD diagnosis, which has clearly been shown to affect outcomes. ${ }^{8}$ Although this has improved in recent years, estimates are still that the delay in diagnosis of IPF is around 2 years. ${ }^{9}$ Undoubtedly, postponement of medical appointments and diagnostic procedures have likely extended the delay in diagnosis of IPF and other ILDs. 
Primary care providers should continue to refer patients with suspected ILD to tertiary referral centers where they can be seen using virtual platforms or in-person consultations where appropriate. Multidisciplinary ILD conferences involving other clinicians, radiologists, and pathologists should continue to be conducted virtually to help minimize delays in diagnosis. Additionally, monitoring of patients with ILD and their response to therapies generally involves serial pulmonary function testing, which has also been restricted during the pandemic. Ongoing care for patients with ILD should include virtual or telephone visits whenever possible, and patientreported symptoms should be used to assess whether current therapies are effective or if changes are needed.

\section{HOW SHOULD COVID-19-POSITIVE PATIENTS WITH UNDERLYING ILD OR SARCOIDOSIS BE TREATED?}

\section{Hospital admission, supportive care}

Because of the risk of severe complications in COVID19 patients with underlying ILD or fibrotic pulmonary sarcoidosis, there should be a low threshold for hospital admission. The current recommendations for treatment of COVID-positive patients involve mostly supportive care including supplemental oxygen, mechanical ventilator support using a lungprotective strategy similar to that used in ARDS, and prone positioning.

\section{Possible drug treatment}

On the basis of preliminary trial data, ${ }^{10}$ the US National Institutes of Health (NIH) now recommends the investigational antiviral agent remdesivir for the treatment of COVID-19 patients hospitalized with severe disease through an FDA emergency use authorization. ${ }^{11}$ Otherwise, patients should be enrolled in ongoing clinical trials regarding potential therapies such as remdesivir, hydroxychloroquine, and/or tocilizumab, and it is strongly recommended that guidance should be elicited from infectious disease physicians regarding use of these medications.

\section{Caution with steroids}

There is evidence that giving steroids to patients with severe acute respiratory syndrome (SARS) and Middle East respiratory syndrome (MERS) results in prolonged viral shedding and worsened clinical outcomes. ${ }^{12}$ Therefore, the World Health Organization (WHO) does not recommend using steroids in COVID-19 except in special cases such as exacer- bation of asthma or chronic obstructive pulmonary disease or septic shock. ${ }^{13}$ Giving antibiotics and steroids in acute exacerbations of ILD has been common practice for many years, but steroid treatment has come under scrutiny as of late, with several trials showing possible harm and increased risk of serious adverse reactions. ${ }^{14,15}$

Histologically, most acute exacerbations of ILD are characterized by diffuse alveolar damage, while others are characterized by organizing pneumonia, alveolar hemorrhage, and unspecific inflammatory changes. Clinical judgment should be used, and steroids could be considered in cases more likely to respond, such as ILD related to exacerbation of underlying connective tissue disease, which may involve more organizing pneumonia or alveolar hemorrhage.

\section{Is immunosuppressive therapy a risk factor?}

Many patients with ILD or sarcoidosis are managed with chronic immunosuppressive therapy. Although the CDC lists it as a risk factor for severe illness with COVID-19, there is evidence from the transplant literature that, unlike other community-acquired viruses such as influenza, adenovirus, or rhinovirus, coronaviruses do not cause more severe disease in immunosuppressed patients, likely because the host innate immune response appears to be the main source of lung tissue damage. ${ }^{16}$ Reports of outbreaks of SARS, MERS, and COVID-19 so far have reported no deaths in transplant recipients, patients receiving chemotherapy, or those receiving other immunosuppressive treatments, at any age..$^{1,17,18}$ As severe illness with COVID-19 is often associated with a hyper-inflammatory state, this has raised the question whether certain immunosuppressive therapies might even be beneficial in these cases. ${ }^{19}$ However, given the absence of strong evidence, it is reasonable to continue to initiate or maintain immunosuppressive therapy in patients with ILD or sarcoidosis according to the current standard of care.

\section{The role of anti-fibrotic therapy in COVID-19}

Pirfenidone and nintedanib are anti-fibrotic drugs that have been used in idiopathic pulmonary fibrosis since 2014, when clinical trials suggested they are both effective in slowing the rate of lung function decline by around $50 \% \cdot{ }^{20,21}$ Based on the recent SENCSIS and INBUILD trials, the use of nintedanib has also now been extended to those with pulmonary fibrosis secondary to systemic sclerosis and in all forms of progressive fibrosing interstitial lung disease (PF-ILD). ${ }^{22,23}$ 
Despite having different mechanisms of action, neither pirfenidone nor nintedanib are considered immunosuppressive therapy and, unless certain complications such as liver or kidney failure arise, there would be no rationale for discontinuation in patients already on medication who contract COVID-19. There has been speculation that given the results of the INBUILD trial, which demonstrated that anti-fibrotic therapy can be effective in pulmonary fibrosis arising from different etiologies, clinical trials involving anti-fibrotic therapy in COVID-19 should be conducted. ${ }^{24}$ In fact, a single center study trial of nintedanib in patients with severe COVID-19 in China is currently being initiated. However, there is no evidence yet supporting initiation of anti-fibrotic therapy in patients with COVID-19.

\section{Consider avoiding mechanical ventilation in acute exacerbations of ILD}

The mortality rate in acute exacerbations of ILD is greater than $50 \% .{ }^{25}$ Initial reports suggested high mortality rates in COVID-19 patients requiring mechanical ventilation. More recent evidence suggests the mortality rate in ventilated COVID-19 patients is somewhere between $16 \%$ and $25 \% .{ }^{26,27}$ Nevertheless, given this added risk to the already high risk of mortality in patients with ILD requiring intubation, strong consideration should be given to avoiding mechanical ventilation and considering palliative measures in a COVID-positive patient with an acute exacerbation of ILD.

\section{PATIENTS WHO DEVELOP COVID-19 RESPIRATORY ILLNESS ARE AT RISK FOR DEVELOPING ILD}

As we are still early in this pandemic, not much is known about the long-term outcomes of COVID-19 respiratory infection. Numerous reports from survivors of SARS and MERS describe long-term lung damage and pulmonary fibrosis, resulting in reduced quality-of-life and significant disability..$^{28}$ In a 1-year follow-up of SARS survivors, $24 \%$ had impaired diffusing capacity for carbon monoxide, and 27.8\% had radiographic evidence of interstitial changes or pulmonary fibrosis, or both..$^{29}$ A 2019 meta-analysis by Sheng at $\mathrm{al}^{30}$ concluded that a number of different viruses are associated with the development of idiopathic pulmonary fibrosis (likely as a trigger rather than the cause). As the pathology and mechanism of infection are similar to those seen in SARS and MERS, one would expect to see similar outcomes of chronic ILD lung disease and reduced pulmonary function in some survivors of COVID-19.

\section{REFERENCES}

1. Yang J, Zheng $Y$, Gou $X$, et al. Prevalence of comorbidities and its effects in patients infected with SARS-CoV-2: a systematic review and meta-analysis. Int J Infect Dis 2020; 94:91-95. doi:10.1016/j. ijid.2020.03.017

2. Centers for Disease Control and Prevention (CDC). Coronavirus disease 2019 (COVID-19): People who are at higher risk for severe illness. Reviewed May 14, 2020. Accessed June 12, 2020. https://www. cdc.gov/coronavirus/2019-ncov/need-extra-precautions/people-athigher-risk.html

3. Zhou $\mathbf{P}$, Yang $\mathbf{X L}$, Wang XG, et al. A pneumonia outbreak associated with a new coronavirus of probable bat origin. Nature 2020; 579(7798):270-273. doi:10.1038/s41586-020-2012-7

4. Xu Z, Shi L, Wang Y, et al. Pathological findings of COVID-19 associated with acute respiratory distress syndrome. Lancet Respir Med 2020; 8(4):420-422. doi:10.1016/S2213-2600(20)30076-X

5. Barton LM, Duval EJ, Stroberg E, Ghosh S, Mukhopadhyay S. COVID-19 autopsies, Oklahoma, USA. Am J Clin Pathol 2020; 153(6):725-733. doi:10.1093/ajcp/aqaa062

6. Kreuter M, Polke M, Walsh SLF, et al. Acute exacerbation of idiopathic pulmonary fibrosis: international survey and call for harmonisation. Eur Respir J 2020; 55(4):1901760. Published April 3, 2020. doi:10.1183/13993003.01760-2019

7. Panselinas $\mathbf{E}$, Judson MA. Acute pulmonary exacerbations of sarcoidosis. Chest 2012; 142(4):827-836. doi:10.1378/chest.12-1060

8. Oldham JM, Noth I. Idiopathic pulmonary fibrosis: early detection and referral. Respir Med 2014; 108(6):819-829. doi:10.1016/j. rmed.2014.03.008

9. Hoyer N, Prior TS, Bendstrup E, Wilcke T, Shaker SB. Risk factors for diagnostic delay in idiopathic pulmonary fibrosis. Respir Res 2019; 20(1):103. Published May 24, 2019. doi:10.1186/s12931-019

10. Beigel JH, Tomashek KM, Dodd LE, et al. Remdesivir for the treatment of Covid-19-preliminary report [published online ahead of print May 22, 2020]. N Engl J Med 2020; NEJMoa2007764. doi:10.1056/NEJMoa2007764

11. National Institutes of Health (NIH). COVID-19 treatment guidelines. What's new in the guidelines. Updated June 11, 2020. Accessed June 12, 2020. https://covid19treatmentguidelines.nih. gov/whats-new/

12. Russell CD, Millar JE, Baillie JK. Clinical evidence does not support corticosteroid treatment for 2019-nCoV lung injury. Lancet 2020; 395(10223):473-475. doi:10.1016/\$0140-6736(20)30317-2

13. World Health Organization. Clinical management of COVID-19. Updated May 27, 2020. Accessed June 12, 2020. https://www. who.int/publications-detail/clinical-management-of-severe-acuterespiratory-infection-when-novel-coronavirus-(ncov)-infection-issuspected

14. Cuerpo S, Moisés J, Hernández-González F, et al. Acute exacerbations of idiopathic pulmonary fibrosis: does clinical stratification or steroid treatment matter? Chron Respir Dis 2019; 16:1479973119869334. doi:10.1177/1479973119869334

15. Marchioni A, Tonelli R, Ball L, et al. Acute exacerbation of idiopathic pulmonary fibrosis: lessons learned from acute respiratory distress syndrome. Crit Care 2018; 22(1):80. doi:10.1186/s13054-018-2002-4

16. D'Antiga L. Coronaviruses and immunosuppressed patients: the facts during the third epidemic. Liver Transpl 2020; 26(6):832-834. doi:10.1002/lt.25756

17. Hui DS, Azhar El, Kim YJ, Memish ZA, Oh MD, Zumla A. Middle East respiratory syndrome coronavirus: risk factors and determinants of primary, household, and nosocomial transmission. Lancet Infect Dis 2018; 18(8):e217-e227. doi:10.1016/S1473-3099(18)30127-0

18. Liang W, Guan W, Chen $\mathbf{R}$, et al. Cancer patients in SARS-CoV-2 infection: a nationwide analysis in China. Lancet Oncol 2020; 21(3):335-337. doi:10.1016/S1470-2045(20)30096-6

19. Mehta P, McAuley DF, Brown M, et al. COVID-19: consider cytokine storm syndromes and immunosuppression. Lancet 2020; 395(10229):1033-1034. doi:10.1016/S0140-6736(20)30628-0

20. King TE Jr, Bradford WZ, Castro-Bernardini S, et al. A phase 3 trial of pirfenidone in patients with idiopathic pulmonary fibrosis. N Engl J 
Med 2014; 370(22):2083-2092. doi:10.1056/NEJMoa1402582

21. Richeldi L, du Bois RM, Raghu G, et al. Efficacy and safety of nintedanib in idiopathic pulmonary fibrosis. N Engl J Med 2014; 370(22):2071-2082. doi:10.1056/NEJMoa1402584

22. Distler O, Highland KB, Gahlemann M, et al. Nintedanib for systemic sclerosis-associated interstitial lung disease. N Engl J Med 2019; 380(26):2518-2528. doi:10.1056/NEJMoa1903076

23. Flaherty KR, Wells AU, Cottin V, et al. Nintedanib in progressive fibrosing interstitial lung diseases. N Engl J Med 2019; 381(18):17181727. doi:10.1056/NEJMoa1908681

24. George PM, Wells AU, Jenkins RG. Pulmonary fibrosis and COVID19: the potential role for antifibrotic therapy [published online ahead of print May 15, 2020]. Lancet Respir Med 2020; S22132600(20)30225-3. doi:10.1016/S2213-2600(20)30225-3

25. Song JW, Hong SB, Lim CM, Koh Y, Kim DS. Acute exacerbation of idiopathic pulmonary fibrosis: incidence, risk factors and outcome. Eur Respir J 2011;37(2):356-363. doi:10.1183/09031936.00159709

26. Ziehr DR, Alladina J, Petri CR, et al. Respiratory pathophysiology of mechanically ventilated patients with COVID-19: a cohort study [published online ahead of print April 29, 2020]. Am J Respir Crit Care Med 2020; 10.1164/rccm.202004-1163LE. doi:10.1164/rccm.202004-1163LE

27. Richardson S, Hirsch JS, Narasimhan M, et al. Presenting characteristics, comorbidities, and outcomes among 5700 patients hospitalized with COVID-19 in the New York city area. JAMA 2020; 323(20):2052-2059. doi:10.1001/jama.2020.6775

28. Ngai JC, Ko FW, Ng SS, To KW, Tong M, Hui DS. The long-term impact of severe acute respiratory syndrome on pulmonary function, exercise capacity and health status. Respirology 2010; 15(3):543-550. doi:10.1111/j.1440-1843.2010.01720.x

29. Hui DS, Wong KT, Ko FW, et al. The 1-year impact of severe acute respiratory syndrome on pulmonary function, exercise capacity, and quality of life in a cohort of survivors. Chest 2005; 128(4):2247-2261. doi:10.1378/chest.128.4.2247

30. Sheng G, Chen $\mathbf{P}$, Wei $Y$, et al. Viral infection increases the risk of idiopathic pulmonary fibrosis: a meta-analysis. Chest 2020; 157(5):1175-1187. doi:10.1016/j.chest.2019.10.032 